\title{
Analyzing Negative Experiences Without Ruminating: The Role of Self-Distancing in Enabling Adaptive Self-Reflection
}

\author{
Özlem Ayduk ${ }^{1 *}$ and Ethan Kross ${ }^{2}$ \\ ${ }^{1}$ University of California, Berkeley \\ 2 University of Michigan, Ann Arbor
}

\begin{abstract}
Both common intuition and findings from multiple areas of research suggest that when faced with distressing experiences, it is helpful to understand one's feelings. However, a large body of research also indicates that people's attempts to make sense of their feelings often backfire, leading them to ruminate and feel worse. In this article, we describe a program of research that focuses on disentangling these seemingly contradictory sets of findings. The research program we describe proposes that psychological distance from the self plays a key role in determining whether people's attempts to understand their feelings lead to adaptive or maladaptive self-reflection. It suggests that people's attempts to understand their feelings often fail because they analyze their feelings from a self-immersed perspective rather than a self-distanced perspective. Empirical evidence from multiple levels of analysis is presented to support this prediction. The basic science and clinical implications of these findings are discussed.
\end{abstract}

Many of us have the intuitive belief that understanding our reactions to negative experiences will empower us, enabling us to gain closure and move on with our lives (e.g., Smith \& Alloy, 2009). However, decades of research examining the implications of selfreflection for affect and behavior have revealed contradictory findings. Whereas some research finds that self-reflection of negative experiences is adaptive, facilitating long-term resolution of distress (e.g., Pennebaker \& Chung, 2007), other research suggests that selfreflection is maladaptive, leading to rumination and the escalation of negative affect (e.g., Nolen-Hoeksema, Wisco, \& Lyubomirsky, 2008). Given these contradictory findings, a key challenge is to determine how people can adaptively analyze negative experiences without ruminating. This manuscript will review findings from a program of research on self-distancing that focuses on addressing this issue.

\section{When Self-Reflection is Adaptive: Benefits of Focusing on Negative Experiences}

A large body of research from multiple areas of psychology has documented the benefits of focusing on and confronting negative experiences for facilitating adaptive coping and psychological adjustment. For example, research has shown that interventions that direct individuals to construct narratives about upsetting events lead to a variety of physical and mental health benefits presumably by leading people to assign meaning, coherence, and structure to their emotions (for reviews see Pennebaker \& Chung, 2007 and Smyth, 1998; also see Wilson \& Gilbert, 2008). Similarly, strategies that lead to changes in the way people cognitively represent distressing events have been 
associated with a host of adaptive outcomes including reductions in emotional and physiological reactivity, improved memory of emotional events, and long-term gains in personal and interpersonal well-being (e.g., Abler, Erk, Herwig, \& Walter, 2007; Lazarus \& Alfert, 1964; Nezlek \& Kuppens, 2008; Ray, Wilhelm, \& Gross, 2008; Resick et al., 2008). The latter findings are especially noteworthy because teaching people how to reconstrue their negative thoughts is a central goal of various forms of cognitive therapy (e.g., Beck, 1970; Ellis, 1962; Linehan, 1993; Teasdale et al., 2002), and central tenet of research on emotion regulation (see Ochsner \& Gross, 2008 for review).

These findings regarding the benefits of focusing on and reconstruing negative experiences are complemented by theory and research arguing that experiential avoidance of negatively valenced information through such strategies as cognitive avoidance, suppression, and distraction hinder rather than facilitate the long-term resolution of distress (e.g., Foa \& Kozak, 1986; Hayes, Wilson, Gifford, Follette, \& Strosahl, 1996). For example, although using distraction to avoid thinking about a negative experience provides people with an immediate reprieve from distress (e.g., Nolen-Hoeksema, 1991), this benefit tends to be short lived because distraction does not allow individuals to directly deal with the cognitive representations that are the source of their negative emotions (CampbellSills \& Barlow, 2007; Kross \& Ayduk, 2008). In fact, people who try to avoid thinking about their negative experiences often become preoccupied with them, thinking about them repetitively rather than getting over them (Cribb, Moulds, \& Carter, 2006; Moulds, Kandris, Starr, \& Wong, 2007). This preoccupation is likely to explain the link between avoidance and various stress reactions, including PTSD symptoms, depression, anxiety, somatic symptoms, and HPA axis reactivity (e.g., Sundin \& Horowitz, 2002). Similarly, expressive suppression also increases the risk for both personal and interpersonal difficulties (Gross \& John, 2003).

\section{When Self-Reflection is Maladaptive: Costs of Focusing on Negative Experiences}

Although the findings reviewed earlier underscore the necessity of confronting rather than avoiding negative thoughts and emotions to fully recover from them, an alternative literature reveals that people's everyday attempts to understand their emotions often backfire. Rumination refers to a process in which people focus repeatedly and passively on what they are feeling and why they are feeling a certain way (e.g., Nolen-Hoeksema et al., 2008; Smith \& Alloy, 2009). Research shows that people do this because they believe that understanding their feelings will improve their mood (e.g., Papageorgiou \& Wells, 2001; Smith \& Alloy, 2009). However, focusing attention on self-relevant negative content increases the accessibility of other negative thoughts and emotions, compromising people's problem-solving abilities and ultimately functions to perpetuate, rather than reduce, negative mood states (Nolen-Hoeksema, 1991; Nolen-Hoeksema et al., 2008). Not surprisingly, rumination is a common key risk factor across many clinical disorders (Harvey, Watkins, Mansell, \& Shafran, 2004).

Putting these findings together, it is clear that long-term, adaptive coping with distress requires individuals to confront negative experiences so they can change the way they think about them. On the other hand, people's attempts to do this often lead to rumination. The question then is: Is there a way that people can analyze their negative experiences adaptively, without ruminating? 


\section{Self-Distancing as an Enabling Mechanism for Adaptive Self-Reflection}

In an attempt to address this issue, we have suggested that psychological distance - a process in which peoples' direct egocentric experience of a stimulus in the here and now is diminished (e.g., Liberman \& Trope, 2008; Mischel \& Rodriguez, 1993) - plays a critical role in enabling people to reflect over negative experiences adaptively (see Kross, 2009 for review). The concept of psychological distance - broadly construed - and its relevance for coping and self-regulation have a long history in both social psychological and clinical research. For example, Mischel's seminal work on delay of gratification indicates that cognitive strategies that function to psychologically distance children from the emotional impact of appetitive stimuli (e.g., abstraction) enhance their ability to forgo immediate gratification for the sake of long-term goals (Mischel \& Rodriguez, 1993; see Mischel \& Ayduk, 2004 for review). More recently, research on construal level theory has shown that psychological distance (whether conceptualized along the dimension of time, space, or social relationships) leads people to adopt broader perspectives on events, helping people to see the 'big-picture' rather than focusing on the concrete details and thus facilitating self-regulation (Fujita, Trope, Liberman, \& Levin-Sagi, 2006; Trope \& Liberman, 2003; see Liberman \& Trope, 2008 for review).

In the clinical domain, Beck identified 'distancing' from one's egocentric thoughts and beliefs as an important precondition for enabling individuals to implement cognitive strategies aimed at alleviating depression (Alford \& Beck, 1997; Beck, 1970). Psychological distancing, although clearly not identical, also overlaps with the concept of 'decentering' common to many 'Third-Wave' forms of cognitive behavioral therapy (e.g., Mindfulness-Based Cognitive Therapy; Acceptance and Commitment Therapy; and Dialectical Behavior Therapy) in that clients are taught to 'step back' from their thoughts and feelings so that they can observe them as 'passing mental events' (Hayes, Strosahl, \& Wilson, 1999; Linehan, 1993; Teasdale et al., 2002; Watkins, Teasdale, \& Williams, 2003).

Drawing from and integrating these different lines of research and viewpoints we reasoned in prior research (Ayduk \& Kross, 2008, 2009; Kross \& Ayduk, 2008, 2009; Kross, Ayduk, \& Mischel, 2005) that to enable people to reflect over negative experiences without ruminating, a strategy was needed to 'distance' them from their feelings so that they could reconstrue them adaptively. We further reasoned that one way of leading people to distance is to manipulate the type of self-perspective they adopt when they focus on understanding their feelings. Specifically, prior research indicates that people are capable of focusing on autobiographic experiences from either a self-immersed perspective, in which they visualize events happening to them through their own eyes or a self-distanced perspective, in which they see themselves in their experience from the perspective of an observer or 'fly on the wall' (e.g., Nigro \& Neisser, 1983; Robinson \& Swanson, 1993).

We reasoned that directing individuals to analyze their feelings from a self-distanced perspective should allow them to focus on, activate, and analyze their feelings, but from a perspective that is sufficiently removed so as to allow them to engage in the kind of reconstrual processes that facilitate adaptive self-reflection. In contrast, we predicted that when people analyze their experiences from a self-immersed perspective - the type of self-perspective people typically adopt when recalling negative autobiographic memories (Nigro \& Neisser, 1983) - they would be more likely to focus on recounting the concrete, emotionally arousing details of their experience (i.e. what happened to me? What did I feel?), and less likely to reconstrue how they feel. In turn, we predicted that this shift in the balance of thought content - more recounting of what happened and less reconstruing of 
one's experience- would lead people who analyze their feelings from a self-immersed perspective to be more vulnerable to rumination.

We tested these predictions in multiple experiments using both single-session and longitudinal designs using a range of outcome variables that span multiple levels of analysis, from self-reported affect to cognitive accessibility measures to cardiovascular indices of distress. In the following sections, we summarize the key findings that have been generated thus far.

\section{Self-Distancing Facilitates Adaptive Self-Reflection: Empirical Evidence}

\section{Self-distancing buffers against short-term emotional reactivity}

Because rumination increases negative affect, one of the most common methods for assessing whether it has occurred is to examine how upset people report feeling after analyzing their emotions (e.g., Nolen-Hoeksema, 1991). Therefore, as a first step toward examining whether self-distancing buffers individuals against rumination, we directed participants to recall an intense anger-related experience from their past and then randomly assigned them to focus on their experience from either a self-immersed perspective ("Now close your eyes... go back to the time and place of the experience... Now see the situation unfold through your own eyes as if it were happening to you all over again') or a self-distanced perspective ("Now close your eyes...go back to the time and place of the experience... Now take a few steps back. Move away from the situation to a point where you can now watch the experience unfold from a distance and see yourself in the event. As you do this, focus on what has now become the distant you... Now watch the situation unfold as if it were happening to the distant you all over again'). Subsequently, participants in both groups were asked to analyze their feelings while maintaining the perspective they were initially told to adopt and then report on their thoughts and emotions. The results indicated that participants in the self-distanced group displayed significantly lower levels of negative affect and reported 'reliving' their recalled experience less than participants in the self-immersed group. Furthermore, the self-distanced group showed lower cognitive accessibility of anger-related thoughts than the immersed group a finding that argues against a simple demand explanation for the effect of self-distancing.

Recent studies have replicated and extended these findings in multiple directions. For example, in one set of studies we demonstrated that self-distancing leads to similar affect buffering effects when participants analyze depression-related experiences (Kross \& Ayduk, 2008; Studies 1 and 2). In another line of work by an independent group of researchers, Gruber, Harvey, and Johnson (2009) demonstrated that directing individuals with bipolar disorder to analyze a recent positive experience from a self-distanced (in comparison with a self-immersed) perspective led them to experience significantly lower levels of positive affect and physiological arousal. These findings are particularly noteworthy because they demonstrate that self-distancing helps down-regulate the intensity of affect regardless of valence, and therefore, may also be a helpful regulatory strategy in disorders that involve excessive positive affect (e.g., bipolar disorder and mania).

\section{Thought content mediates the effect of self-distancing on short-term emotional reactivity}

The findings described previously demonstrate that self-distancing leads to reductions in emotional reactivity following the analysis of personally relevant emotional events. But how does distancing lead to these emotional changes? Our prediction was that 
analyzing negative feelings from a self-distanced perspective should lead people to focus less on recounting the concrete, emotionally arousing details of their experiences and more on reconstruing the event and their reactions to it in ways that promote insight and closure. In turn, we hypothesized that this shift in thought content - less recounting and more reconstruing - would lead people to feel less upset after analyzing their feelings.

We tested this prediction in multiple studies by asking participants to describe in writing the stream of thoughts that flowed through their minds as they analyzed their feelings (Kross et al., 2005; Study 2; Kross \& Ayduk, 2008, Studies 1 and 2). Judges blind to condition then coded these essays for the degree to which they contained recounting statements (i.e., descriptions of the specific chain of events and emotions participants experienced during the event) and reconstruing statements (i.e., descriptions of new realizations about or changes in the way participants understood the causes underlying their feelings).

Across studies we consistently find three key results. First, all participants engage in more recounting than reconstruing. This finding is noteworthy because it indicates that all people, regardless of the type of self-perspective they adopt while analyzing their feelings, focus on the emotionally arousing features of their recalled experience rather than avoid them. Second, despite the fact that everyone focuses more on recounting what happened, participants in the self-distancing group engage in relatively less recounting and relatively more reconstruing than participants in the self-immersion group. Finally, this shift in thought content displayed by the self-distanced group - less recounting and more reconstruing (operationalized as the difference score between the two variables) - leads them to display significantly lower levels of negative affect. In statistical terms, thought content mediates the effect of the self-perspective manipulations on negative affect.

We have tested alternative mediation models across multiple studies to demonstrate that it is the balance between these two processes - less recounting and more reconstruing that accounts for the affect regulatory effects of self-distancing. For example, we have demonstrated that including either recounting alone (e.g., Self-Perspective $\rightarrow$ Recounting $\rightarrow$ Affect) or reconstrual alone (e.g., Self-Perspective $\rightarrow$ Reconstruing $\rightarrow$ Affect) does not provide a better fit for the data than the model described earlier, in which the difference between these two variables is included as the mediator. We have also tested a model that simultaneously includes both recounting and reconstruing as separate mediators and likewise have not found this to provide a better fit for the data than when the difference between these variables is used as the mediator (see Kross \& Ayduk, 2008 for details; also see Grossmann \& Kross, forthcoming). Collectively, these findings indicate that it is the balance between recounting and reconstrual rather that each mechanism alone that accounts for the effect of self-distancing on affect.

\section{The buffering effect of self-distancing extends to autonomic nervous system indices of distress}

To extend the impact of self-distancing beyond subjective reports of distress (which are subject to self-report bias and demand), we have also focused on physiological markers of distress. Rumination delays the amount of time it takes people to physiologically recover from negative experiences by leading individuals to continually brood over their feelings (Brosschot, Gerin, \& Thayer, 2006; Glynn, Christenfeld, \& Gerin, 2002). A number of studies have shown that increased physiological recovery times of this sort are a key risk factor for cardiovascular disease (e.g., McEwen, 1998) - a finding that has led a number of researchers to suggest that rumination may enhance cardiac 
disease vulnerability through its effects on recovery (Brosschot et al., 2006). Therefore, to the extent that analyzing negative experiences from a self-distanced perspective attenuates rumination, we hypothesized that it should attenuate cardiovascular reactivity when participants are explicitly directed to analyze their emotions, while also facilitating recovery.

We tested this hypothesis by continuously recording participants' blood pressure while they analyzed their emotions about an anger-eliciting event and during a recovery period approximately 20 minutes after the experimental manipulations were administered (Ayduk \& Kross, 2008). The results showed that participants in the self-distanced group displayed significantly lower levels of blood pressure reactivity (elevations relative to baseline) compared to the self-immersed group both during the analysis and the recovery periods of the experiment. These findings extend the beneficial effects of self-distancing to physiological indices of stress that have relevance for physical health outcomes. Furthermore, they demonstrate that the beneficial effect of self-distancing is not restricted to the thin slice of time during which participants are told to analyze their emotions; rather it has implications for how people feel over time.

\section{Short- and long-term effects of self-distancing in comparison with distraction}

We have also compared the effectiveness of self-distancing against distraction differentiating between the short- and long-term effectiveness of these strategies. Distraction has been used as the gold-standard technique for reducing emotional reactivity in rumination research (Nolen-Hoeksema et al., 2008). However, as discussed earlier because distraction is not instrumental in altering the cognitive representations that are the source of negative emotions, its usefulness is likely to be limited to the short period of time during which it is actively used.

In contrast, because analyzing negative emotions from a self-distanced perspective directly influences how people construe the meaning of their experience, it should help people react less strongly to negative experiences not only when it is actively used (i.e., in the short-term) but also in response to subsequent reminders of these events, displaying long-term benefits. In other words, self-distancing should be as effective as distraction in the short-term but more effective than distraction in the long-term for reducing emotional reactivity. We tested these hypotheses in a series of studies.

Short-term effects. First, in two studies we pitted the short-term effect of self-distanced analyses against distraction by asking participants to recall a depression experience and then randomly assigning them to a self-immersed, self-distanced, or distraction condition (Kross \& Ayduk, 2008; Study 1; Kross \& Ayduk, 2008; Study 2). The instructions for the self-distancing and self-immersion manipulations followed the standard protocol we described earlier. In the distraction condition, participants were presented with a series of nonemotional facts (e.g., Switzerland is in Europe; Pencils are made of lead) and were asked to think about each fact for 8 seconds, following prior research (e.g., Nolen-Hoeksema \& Morrow, 1991). We found that distraction and self-distancing led to significantly lower levels of emotional reactivity relative to self-immersion in both studies. However, distraction and self-distancing did not differ significantly from each other in either experiment. These findings help gauge the relative strength of self-distancing for attenuating emotional reactivity by demonstrating that it is as effective as distraction for reducing negative affect in the short-term. 
Long-term effects. We compared the long-term benefits of self-distancing and distraction by including a longitudinal component to one of our studies described earlier in which participants returned to the laboratory either 1-day or 1-week after the experimental manipulations were administered (Kross \& Ayduk, 2008, Study 2). During this second testing session, participants were asked to recall the experience they thought about during the first session once again. They were then cued to think about their deepest thoughts and feelings regarding that event. Importantly, participants were not given any specific instructions regarding what type of self-perspective they should adopt while thinking about their experience during the second session.

Regardless of the time lag between the two sessions, participants in the self-distancing group displayed lower levels of negative affect at Time 2 than participants in the self-immersion and distraction groups. Furthermore, when participants were asked how often they thought about the event they recalled over the time period separating the two sessions, those in the self-immersion and distraction groups reported higher levels of repetitive thought than those in the self-distanced group. In summary, self-distancing allowed individuals to reconstrue the distress eliciting features of negative experiences in a way that attenuated future negative emotional reactions and recurring thoughts, both of which are hallmark features of ruminative processing (Watkins, Moberly, \& Moulds, 2008).

\section{Beneficial effects of self-distancing extend to dysphoric populations}

The studies reviewed thus far were conducted with normal healthy populations. However, it is important to examine whether there is continuity or discontinuity in the emotion regulatory processes related to self-distancing across clinical and nonclinical populations to establish the boundary conditions that determine how self-distancing operates. As a first step toward addressing this question, we (Kross \& Ayduk, 2009) pooled data from five published studies (Ayduk \& Kross, 2008; Kross et al., 2005; Studies 1 \& 2; Kross \& Ayduk, 2008; Studies 1 \& 2), which also included unanalyzed Beck Depression Inventory (BDI; Beck, Ward, Mendelson, Mock, \& Erbaugh, 1961) data. Pooling data across these studies provided us with a sample large enough to reasonably represent individuals who scored low vs. high on the BDI, thus allowing us to begin to examine how the findings described earlier generalize to dysphoric populations.

Two key findings emerged from these analyses. First, although self-distancing had a beneficial effect on reducing negative affect at the group level, its effectiveness increased linearly with depressive symptoms. That is, the more dysphoric participants were, the more they benefitted from analyzing their feelings from a self-distanced perspective compared to a self-immersed perspective. Because depression is characterized by the tendency to magnify emotional responses to stressful experiences (e.g., Abramson \& Alloy, 2006; Beck, Rush, Shaw, \& Emery, 1979), this finding suggests that selfdistancing may be more useful for those who are particularly vulnerable to heightened negative affect and rumination. Nevertheless, perspective manipulations influenced the balance between recounting and reconstruing similarly regardless of depressive symptoms and the same causal pathway (lower recounting, higher reconstruing) explained the effect of self-distancing on lower emotional reactivity for individuals high and low in depression.

Overall, these findings demonstrate that rather than increasing emotional reactivity of dysphoric individuals, self-distancing benefitted them even more than it did nondysphoric individuals. As we discuss later, however, a key need for future research is to address this 
issue directly by examining how these perspective manipulations operate in clinically depressed individuals.

\section{Individual differences in spontaneous self-distancing}

The findings reviewed earlier demonstrate that directing people to analyze negative experiences from a self-distanced perspective in the laboratory leads to a variety of physical and mental health benefits. However, to establish the processes and consequences of self-distancing as general principles of behavior, it is critical to examine whether these experimental findings generalize to how self-distancing impacts affect and rumination when it is used spontaneously in people's everyday attempts to cope with negative experiences.

We have begun to address this issue by adapting our standard experimental protocol to assess individual differences in spontaneous self-distancing. More specifically, participants are asked recall and analyze their emotions about a recent negative experience and then indicate the degree to which they adopted a self-immersed vs. a self-distanced perspective on a likert scale $(1=$ predominantly immersed, $7=$ predominantly distanced; see Ayduk \& Kross, 2010). Preliminary findings indicate that spontaneous self-distancing is associated with a remarkably similar profile of processes and outcomes as experimentally manipulated self-distancing. Briefly, higher levels of spontaneous self-distancing during analysis of negative past experiences predict lower emotional reactivity, and this association is mediated by thought content (i.e., less recounting and more reconstrual). Similar to experimental findings, spontaneous self-distancing is associated with lower cardiovascular reactivity during analysis of stressful experiences as well as a recovery period. Finally, spontaneous self-distancing predicts significant reductions in emotional reactivity to the same eliciting event, and lower levels of intrusive ideation longitudinally (over a 7-week period), indicating that at least in nonclinical populations, the spontaneous use of this strategy is associated with markers of adaptive self-reflection.

Evidence is also emerging on the trait correlates of self-distancing, in particular with respect to trait rumination and reappraisal. Consistent with the idea that self-immersion makes people vulnerable to rumination, whereas self-distancing protects against it, our preliminary findings indicate that spontaneous self-distancing is negatively associated with trait rumination (Ayduk \& Kross, 2010). However, spontaneous distancing is not significantly correlated with individual differences in either suppression or reappraisal, as measured by the Emotion Regulation Questionnaire (EQR, Gross \& John, 2003). At first, the latter finding may seem contradictory to our experimental findings demonstrating how adopting a self-distanced perspective leads to cognitive change. However, we view self-distancing as one of many different cognitive tactics people can use to reappraise a negative event and, therefore, are not surprised to see a lack of systematic association with the reappraisal subscale of the ERQ, which is silent about the specific strategies one can adopt in the service of reappraisal.

Despite these initial findings on the link between spontaneous distancing and markers of adaptive self-reflection, we do not yet know whether individual differences in the use of this strategy function as a disposition - whether these differences are stable across time and different types of experiences (e.g., positive and negative in valence) and show meaningful relationships to other, theoretically relevant personality constructs and emotion regulation styles. We also do not know whether individual differences in spontaneous self-distancing are ability- or motivation based. These questions await further research. 


\section{Current and Future Research Directions}

The findings outlined in the previous section raise a number of interesting questions for future research. In this section, we focus on two issues that we feel are particularly important, which current research is beginning to address.

\section{Clinical implications and boundary conditions}

An important set of questions raised by the present research concerns the clinical implications of analyzing negative experiences from a self-distanced perspective. Although recent findings indicate that self-distancing buffers dysphoric individuals against elevated negative affect (Kross \& Ayduk, 2009), dysphoria is not equivalent to clinical depression. Depression is characterized by increased abstract thinking and particularly, by an overgeneralization bias (i.e., categorical representations that summarize repeated experiences) in organization of autobiographic memory (e.g., Watkins \& Moulds, 2007). Some work suggests that because analyzing emotions and adoption of a third-person perspective both elicit abstract thought on their own, their combination should be even more maladaptive particularly in the context of clinical depression (e.g., Kuyken \& Moulds, 2009; Watkins et al., 2008). However, as we discussed earlier, distancing -as a broadly defined construct - has long been recognized as a therapeutic precondition in cognitive behavior therapy of depression and more recently in mindfulness-based cognitive behavior therapies predicting treatment success (e.g., Teasdale et al., 2002). Therefore, these contradictory predictions about the implications of analyzing negative experiences from a self-distanced perspective in clinical depression need to be reconciled in future research.

In addition to depression, the boundary conditions for self-distancing also need to be examined with respect to other disorders, particularly social phobia and post-traumatic stress disorder (PTSD). People with social phobia tend to visualize and recall memories of public situations that are highly anxiety provoking from an observer perspective (e.g., Coles, Turk, Heimberg, \& Fresco, 2001) and patients with PTSD tend to do the same with respect to trauma memories (e.g., Berntsen, Willert, \& Rubin, 2003). These findings suggest that the persistent use of an observer perspective in these clinical populations may serve an avoidance function and undermine adaptive self-reflection over time (although see Resick et al., 2008 for an alternative account). As we discussed elsewhere (Ayduk \& Kross, 2010), however, it is noteworthy that existing clinical work on PTSD and social phobia focus on how the perspective people adopt at memory recall influences well-being and the course of psychopathology. In contrast, our research focuses on the type of self-perspective people adopt as they analyze their memories. These two different stages of information processing - recall vs. analysis, are characterized by substantive differences in that memory recall involves retrieving a memory from longterm memory, whereas analyzing memories involves elaborating on what has been retrieved. It is possible that people who self-distance at recall do so to completely avoid activating their emotions, leaving them vulnerable to the kinds of long-term problems that prior research on PTSD has documented. In contrast, people who self-distance while trying to understand their feelings may be recruiting this process to serve a different function - to enable them to reconstrue their feelings without becoming overwhelmed by negative affect (see Resick et al., 2008 for a consistent rationale). According to this reasoning whether self-distancing is helpful vs. harmful may well depend on why people activate this process (i.e., to avoid focusing on their feelings or to be able to focus on them without becoming overwhelmed) and when they do it 
(i.e., during recall, during analysis, or during both periods) and should be investigated in future research.

One last issue to consider with respect to the boundary conditions for the adaptiveness of self-distancing concerns its use in regulating positive affect. As described earlier, analyzing positive experiences from a self-distanced perspective reduces the intensity of positive affect in individuals diagnosed with bipolar disorder, which is characterized by excessive positive affect (Gruber et al., 2009). However, increased positive affect is predictive of adaptive outcomes in normal populations (e.g., Cohen \& Pressma, 2006; Lyubomirsky, King, \& Diener, 2005). Therefore, outside of the context of bipolar disorder, it may be more adaptive to self-immerse than to self-distance when focusing on positive experiences. It is also possible that the combination of self-distancing from negative and self-immersing in positive experiences is the strategy profile that maximizes mental health benefits. These interesting questions too await future research.

\section{The neural correlates of self-distancing}

Finally, as research continues it will be important to examine the specific patterns of neural activity that underlie and distinguish adaptive and maladaptive forms of self-reflection. Addressing this issue is critical to linking findings about the cognitive operations that facilitate vs. impair people's ability to adaptively cope with negative experiences with the physiological substrates that underlie these different forms of self-reflection. It is also important for linking researchers who are interested in the role that self-reflection plays in distress, but approach the issue from different perspectives (e.g., psychiatry, biochemistry, psychologists, etc).

In this vein, in recent work we have begun to examine the patterns of neural activity that underlie cognitive strategies that function to psychologically distance people from their emotions while reflecting on negative autobiographic memories. For example, in one recent study Kross and colleagues (Kross, Davidson, Weber, \& Ochsner, 2009) cued participants to reflect over a series of painful autobiographic experiences while they were in the fMRI scanner. On some trials, participants were directed to focus on the concrete sensations they experienced while thinking about their memory (i.e., conceptually similar to the recounting that occurs during self-immersion in the studies reviewed earlier). On other trials, participants were directed to focus on their feelings as passing mental events that were psychologically distant from the self and did not control them (i.e., conceptually similar to the reconstruing that occurs during self-distancing in the studies reviewed earlier).

The results of this study indicated that when participants engaged in the distancing strategy they displayed lower levels of self-report negative affect and reduced levels of activation in brain regions involved in self-referential processing (e.g., medial prefrontal cortex, precuneus, posterior cingulated cortex; e.g., Northoff et al., 2006) and emotional processing (e.g., subgenual anterior cingulated cortex, BA25; e.g., Ressler \& Mayberg, 2007) compared to when they engaged in the immersion strategy. It is noteworthy that recent work has implicated increased levels of activity in the subgenual anterior cingulate cortex in clinical depression (e.g., Greicius et al., 2007; Ressler \& Mayberg, 2007. Therefore, these findings provide important preliminary evidence indicating that strategies that function to distance people as they reflect on personally relevant negative experiences modulate activity in brain regions that have been shown to play a role in emotion regulation and in rumination in prior research. However, although conceptually similar, the manipulations used in the Kross et al.'s (2009) study did not directly correspond to the 
standard self-perspective manipulations. Therefore, a more direct examination of the neural substrates associated with shifts in self-perspective remains to be addressed in future work.

\section{Conclusions}

Research examining the role that different types of self-reflective processes play in facilitating vs. undermining peoples' capacity to adaptively cope with negative life experiences have surged in recent years (Joormann, Dkane, \& Gotlib, 2006; Trapnell \& Campbell, 1999; Treynor, Gonzalez, \& Nolen-Hoeksema, 2003). In one such program of research, we have shown how subtle shifts in the type of self-perspective people adopt as they focus on negative experiences - shifts in perspective that lead people to become less 'immersed' and more 'distanced' from their feelings - can powerfully influence whether self-reflection processes result in adaptive or maladaptive outcomes. Clearly, many important questions remain concerning the role self-distancing plays in mental health - questions that we hope to see addressed in the future, and which are necessary to answer to build a cumulative understanding of the role that psychological distance plays in self-control and emotional health.

\section{Acknowledgment}

Özlem Ayduk and Ethan Kross contributed equally to the preparation of this article.

\section{Short Biographies}

Özlem Ayduk's research is located at the intersection of social, personality, and developmental psychology, and she has authored or coauthored papers in these areas for Journal of Personality and Social Psychology, Psychological Science, Journal of Experimental Social Psychology, Personality and Social Psychology Bulletin, Journal of Research in Personality, Emotion, and Social Development among others. Current research focuses on sensitivity to rejection and attentional control as social-cognitive mediators of adjustment, on the role of self-distancing in facilitating adaptive emotion regulation, and on the social and biological precursors of children's self-regulatory competencies as well as their consequences for life long development. Before coming to University of California, Berkeley, where she presently teaches, Ayduk was a post-doctoral scientist at the Columbia University. She holds a BA in Psychology and Political Science from Bogazici University, Istanbul, Turkey, and a PhD in Psychology from the Columbia University in the city of New York.

Ethan Kross's research is located at the intersection of social-personality, clinical and affective neuroscience research. He has authored or coauthored papers in these areas for Journal of Personality and Social Psychology, Psychological Science, Personality and Social Psychology Bulletin, Biological Psychiatry, Journal of Cognitive Neuroscience, Journal of Research in Personality, Annals of the New York Academy of Sciences, Behaviour Research and Therapy among others. Current research focuses on shedding light on how people can adaptively control emotions and impulses that undermine their goals and compromise their health. He adopts an integrative approach spanning multiple levels of analysis (social-cognition, behavior, neuroscience, ANS) to address these issues, and focuses on a variety of populations (e.g., normal healthy individuals, children, individuals with major depression and bipolar disorder). Before coming to the University of Michigan, Ann Arbor, where he is an Assistant Professor in Social Psychology, Kross was a postdoctoral scientist at Columbia 
University. He holds a BA in Psychology from the University of Pennsylvania in Philadelphia, and a PhD in Psychology from Columbia University in the city of New York.

\section{Endnote}

^ Correspondence address: Özlem Ayduk, Department of Psychology, 3210 Tolman Hall, University of California, Berkeley, CA 94720, USA. Email: ayduk@berkeley.edu

\section{References}

Abler, B., Erk, S., Herwig, U., \& Walter, H. (2007). Anticipation of aversive stimuli activates extended amygdala in unipolar depression. Journal of Psychiatric Research, 41, 511-522.

Abramson, L. Y., \& Alloy, L. B. (2006). Cognitive vulnerability to depression: Current status and developmental origins. In T. E. Joiner, J. S. Brown \& J. Kistner (Eds.), The Interpersonal, Cognitive, and Social Nature of Depression (pp. 83-100). Mahwah, NJ: Erlbaum.

Alford, B. A., \& Beck, T. A. (1997). The Integrative Power of Cognitive Therapy. New York: Guilford Press.

Ayduk, O., \& Kross, E. (2008). Enhancing the pace of recovery: Self-distanced analysis of negative experiences reduces blood pressure reactivity. Psychological Science, 19, 229-231.

Ayduk, O., \& Kross, E. (2009). Asking 'why' from a distance facilitates emotional processing: A reanalysis of Wimalaweera and Moulds (2008). Behaviour Research and Therapy, 47, 88-92.

Ayduk, O., \& Kross, E. (2010). From a distance: Implications of spontaneous self-distancing for adaptive self-reflection. Journal of Personality \& Social Psychology, 98, 809-829.

Beck, A. T. (1970). Cognitive Therapy. Nature and relation to Behavior Therapy. Behavior Therapy, 1, $184-200$.

Beck, A. T., Rush, A. J., Shaw, B. F., \& Emery, G. (1979). Cognitive Therapy of Depression, New York: Guilford.

Beck, A. T., Ward, C. H., Mendelson, M., Mock, J., \& Erbaugh, J. (1961). An inventory for measuring depression. Archives of General Psychiatry, 4, 561-571.

Berntsen, D., Willert, M., \& Rubin, D. C. (2003). Splintered memories or vivid landmarks? qualities and organization of traumatic memories with and without PTSD. Applied Cognitive Psychology, 17, 675-693.

Brosschot, J. F., Gerin, W., \& Thayer, J. F. (2006). The perseverative cognition hypothesis: A review of worry, prolonged stress-related physiological activation, and health. Journal of Psychosomatic Research, 60, 113-124.

Campbell-Sills, L., \& Barlow, D. H. (2007). Incorporating emotion regulation into conceptualizations and treatments of anxiety and mood disorders. In J. J. Gross (Ed), Handbook of Emotion Regulation (pp. 542-560). NY, NY: Guilford Press.

Cohen, S., \& Pressma, S. D. (2006). Positive affect and health. Current Directions in Psychological Science, 15, 122-125.

Coles, M. E., Turk, C. L., Heimberg, R. G., \& Fresco, D. (2001). Effects of varying levels of anxiety within social situations: Relationship to memory perspective and attributions in social phobia. Behavior Research \& Therapy, 39 , 651-665.

Cribb, G., Moulds, M. L., \& Carter, S. (2006). Rumination and experiential avoidance in depression. Behaviour Change, 23, 165-176.

Ellis, A. (1962). Reason and Emotion in Psychotherapy. New York: Lyle Stuart.

Foa, E. B., \& Kozak, M. J. (1986). Emotional processing of fear: Exposure to corrective information. Psychological Bulletin, 99, 20-35.

Fujita, K., Trope, Y., Liberman, N., \& Levin-Sagi, M. (2006). Construal levels and self-control. Journal of Personality and Social Psychology, 90, 351-367.

Glynn, L. M., Christenfeld, N., \& Gerin, W. (2002). The role of rumination in recovery from reactivity: Cardiovascular consequences of emotional states. Psychosomatic Medicine, 64, 714-726.

Greicius, M. D., Flores, B. H., Menon, V., Glover, G. H., Solvanson, H. B., Kenna, H., et al. (2007). Restingstate functional connectivity in major depression: Abnormally increased contributions from subgenual cingulate cortex and thalamus. Biological Psychiatry, 62, 429-437.

Gross, J. J., \& John, O. (2003). Individual differences in two emotion regulation processes: Implications for affect, relationships, and well-being. Journal of Personality and Social Psychology, 85, 348-362.

Grossmann, I., \& Kross, E. (forthcoming). The impact of culture on adaptive vs. maladaptive self-reflection. Psychological Science.

Gruber, J., Harvey, A., \& Johnson, S. (2009). Reflective and ruminative processing of positive emotional memories in bipolar disorder and healthy controls. Behavior Research \& Therapy, 47, 697-704.

Harvey, A. G., Watkins, E. R., Mansell, W., \& Shafran, R. (2004). Cognitive Behavioral Processes Across Psychological Disorders: A Transdiagnostic Approach to Research and Treatment. Oxford, UK: Oxford University Press. 
Hayes, S. C., Wilson, K. G., Gifford, E. V., Follette, V. M., \& Strosahl, K. (1996). Experiential avoidance and behavioral disorders: A functional dimensional approach to diagnosis and treatment. Journal of Consulting and Clinical Psychology, 64(6), 1152-1168.

Hayes, S. C., Strosahl, K. D., \& Wilson, K. G. (1999). Acceptance and Commitment Therapy: An Experiential Approach to Behavior Change. New York, NY, US: Guilford Press.

Joormann, J., Dkane, M., \& Gotlib, I. H. (2006). Adaptive and Maladaptive Components of Rumination? Diagnostic Specificity and Relation to Depressive Biases Behavior Therapy, 37, 269-280.

Kross, E. (2009). When self becomes other. Towards an integrative understanding of the processes distinguishing adaptive self-reflection from rumination. In O. Vilarroya, S. Altran, A. Navarro, K. Ochsner \& A. Tobeña (Eds.), Values, Empathy, and Fairness Across Social Barriers. (pp. 35-40). New York, NY, US: New York Academy of Sciences.

Kross, E., \& Ayduk, O. (2008). Facilitating adaptive emotional analysis: Short-term and long-term outcomes distinguishing distanced-analysis of negative emotions from immersed-analysis and distraction. Personality and Social Psychology Bulletin, 34, 924-938.

Kross, E., \& Ayduk, O. (2009). Boundary conditions and buffering effects: Does depressive symptomatology moderate the effectiveness of distanced-analysis on facilitating adaptive self-reflection? Journal of Research in Personality, 43, 923-927.

Kross, E., Ayduk, O., \& Mischel, W. (2005). When asking “why” doesn't hurt: Distinguishing reflective processing of negative emotions from rumination. Psychological Science, 16, 709-715.

Kross, E., Davidson, M., Weber, J., \& Ochsner, K. (2009). Coping with emotions past: The neural bases of regulating affect associated with negative autobiographical memories. Biological Psychiatry, 65, 361-366.

Kuyken, W., \& Moulds, M. L. (2009). Remembering as an observer: How is autobiographical memory retrieval vantage perspective linked to depression? Memory, 17, 624-634.

Lazarus, R. S., \& Alfert, E. (1964). Short-circuiting of threat by experimentally altering cognitive appraisal. Journal of Abnormal \& Social Psychology, 69, 195-205.

Liberman, N., \& Trope, Y. (2008). The psychology of transcending the here and now. Science, 322, $1201-1205$.

Linehan, M. (1993). Skills Training Manual for Treating Borderline Personality Disorder. New York: Guilford Press.

Lyubomirsky, S., King, L., \& Diener, D. (2005). The benefits of frequent positive affect: Does happiness lead to success? Psychological Bulletin, 131, 803-855.

McEwen, B. S. (1998). Protective and damaging effects of stress mediators. New England Journal of Medicine, 338, 171-179.

Mischel, W., \& Ayduk, O. (2004). Willpower in a cognitive-affective processing system: The dynamics of delay of gratification. In R. Baumeister \& K. Vohs (Eds.), Handbook of Self-Regulation: Research, Theory, and Applications (pp. 99-129). New York: Guilford.

Mischel, W., \& Rodriguez, M. L. (1993). Psychological distance in self-imposed delay of gratification. In R. R. Cocking \& K. A. Renninger (Eds.), Based on Material Presented at a Columbia University Lecture by W. Mischel in Nov 1989 (pp. 109-121). Hillsdale, NJ, England: Lawrence Erlbaum Associates, Inc.

Moulds, M. L., Kandris, E., Starr, S., \& Wong, A. C. M. (2007). The relationship between rumination, avoidance and depression in a non-clinical sample. Behaviour Research and Therapy, 45, 251-261.

Nezlek, J. B., \& Kuppens, P. (2008). Regulating positive and negative emotions in daily life. Journal of Personality, 76, 561-579.

Nigro, G., \& Neisser, U. (1983). Point of view in personal memories. Cognitive Psychology, 15, 467-482.

Nolen-Hoeksema, S. (1991). Responses to depression and their effects on the duration of depressive episodes. Journal of Abnormal Psychology, 100, 569-582.

Nolen-Hoeksema, S., \& Morrow, J. (1991). A prospective study of depression and posttraumatic stress symptoms after a natural disaster: The 1989 Loma Prieta earthquake. Journal of Personality and Social Psychology, 61, $115-121$.

Nolen-Hoeksema, S., Wisco, B. E., \& Lyubomirsky, S. (2008). Rethinking rumination. Perspectives on Psychological Science, 3, 400-424.

Northoff, G., Heinzel, A., de Greck, M., Bermpohl, F., Dobrowolyn, H., \& Panskepp, J. (2006). Self-referential processing in our brain-A meta-analysis of imaging studies on the self. Neuroimage, 31, 440-457.

Ochsner, K. N., \& Gross, J. J. (2008). Cognitive emotion regulation: Insights from social cognitive and affective neuroscience. Current Directions in Psychological Science, 17, 153-158.

Papageorgiou, C., \& Wells, A. (2001). Metacognitive beliefs about rumination in recurrent major depression. Cognitive and Behavioral Practice, 8, 160-164.

Pennebaker, J. W., \& Chung, C. K. (2007). Expressive writing, emotional upheavals, and health. In H. Friedman \& R. Silver (Eds.), Handbook of Health Psychology (pp 263-284). New York: Oxford University Press.

Ray, R. D., Wilhelm, F. H., \& Gross, J. J. (2008). All in the mind's eye? Anger rumination and reappraisal Journal of Personality and Social Psychology, 94, 133-145.

Resick, P. A., Galovski, T. E., Uhlmansiek, M. O., Scher, C. D., Clum, G. A., \& Young-Xu, Y. (2008). A randomized clinical trial to dismantle components of cognitive processing therapy for posttraumatic stress disorder in female victims of interpersonal violence. Journal of Consulting and Clinical Psychology, 76, 243-258. 


\section{Self-distancing}

Ressler, K., \& Mayberg, H. (2007). Targeting abnormal neural circuits in mood and anxiety disorders: From the laboratory to the clinic. Nature Neuroscience, 45, 651-660.

Robinson, J. A., \& Swanson, K. K. (1993). Field and observer modes of remembering. Memory, 1, $169-184$.

Smith, J. M., \& Alloy, L. B. (2009). A roadmap to rumination: A review of the definition, assessment, and conceptualization of this multifaceted construct. Clinical Psychology Review, 29, 116-128.

Smyth, J. M. (1998). Written emotional expression: Effect sizes, outcome types, and moderating variables. Journal of Consulting and Clinical Psychology, 66, 175-184.

Sundin, E. C., \& Horowitz, M. J. (2002). Impact of event scale: Psychometric properties. British Journal of Psychiatry, 180, 205-209.

Teasdale, J. D., Moore, R. G., Hayhurst, H., Pope, M., Williams, S., \& Segal, Z. (2002). Metacognitive awareness and prevention of relapse in depression: Empirical evidence. Journal of Consulting and Clinical Psychology, 70, 275-287.

Trapnell, P. D., \& Campbell, J. D. (1999). Private self-consciousness and the five-factor model of personality: Distinguishing rumination from reflection. Journal of Personality and Social Psychology, 76, 284-304.

Treynor, W., Gonzalez, R., \& Nolen-Hoeksema, S. (2003). Rumination reconsidered: A psychometric analysis. Cognitive Therapy and Research, 27, 247-259.

Trope, Y., \& Liberman, N. (2003). Temporal Construal. Psychological Review, 110, 403-421.

Watkins, E. R., Moberly, N. J., \& Moulds, M. L. (2008). Processing mode causally influences emotional reactivity: Distinct effects of abstract versus concrete construal on emotional response. Emotion, 8, 364-378.

Watkins, E., \& Moulds, M. L. (2007). Reduced concreteness of rumination in depression: A pilot study. Personality and Individual Differences, 43, 1386-1395.

Watkins, E., Teasdale, J. D., \& Williams, R. M. (2003). Contextual questions prevent mood primes from maintaining experimentally induced dysphoria. Cognition \& Emotion, 17, 455-475.

Wilson, T. D., \& Gilbert, D. T. (2008). Explaining away: A model of affective adaptation. Perspectives on Psychological Science, 3, 370-386. 